\title{
A Comparative Histopathological Effect of Caffeinated and Decaffeinated Coffee on the Histomorphology of the Kidney of Adult Male Albino Wistar Rats
}

\author{
${ }^{1}$ Umoh, I. U. and ${ }^{2}$ Jimmy, E. O. \\ ${ }^{I}$ Department of Anatomy, Faculty of Basic Medical Sciences, University of Uyo, Uyo, Nigeria. \\ ${ }^{2}$ Department of Physiology, Faculty of Basic Medical Sciences, University of Uyo, Uyo, Nigeria.
}

\begin{abstract}
The comparative effect of caffeinated and decaffeinated coffee on the histomorphology of the kidney of male albino Wistar rats was investigated. Thirty-five (35) male albino Wistar rats (weighing between 135 $185 \mathrm{~g}$ ) were grouped into seven (7) groups with 5 rats in each group. Group 1 served as the control while Groups 2 to 7 were the test groups. Caffeinated coffee at doses of 5mg, 10mg and 15mg per kg body weight of rats were administered orally to Groups 2, 3, 4 respectively. 5mg, 10mg, $15 \mathrm{mg}$ per $\mathrm{kg}$ body weight of decaffeinated coffee were administered orally to Groups 5, 6, 7. After 28 days of administration of the coffee, the animals were sacrificed, kidneys were harvested, fixed, processed and cut at 5 microns, stained using Hematoxylin and Eosin ( $H$ and $E$ technique) and observed for histopathological alterations under light microscope. Results showed numerous tubular degeneration, vascular degeneration, tubular necrosis, glomerular inflammation and loss of epithelial lining in the test groups when compared to the control Group 1. Statistically, the body weight of the rats was observed to be significantly different in coffee treated groups ( $p$ $<0.05)$ when compared to the control. In conclusion, caffeinated coffee has severe effect on the kidney of male albino Wistar rats but is significantly reduced with decaffeinated coffee.
\end{abstract}

Keywords: Caffeinated Coffee, Decaffeinated Coffee, Kidney, Tubular Necrosis, glomerular inflammation.

\section{Introduction}

The kidneys are bean-shaped organs that serve essential excretory roles such as removal of excess organic molecules from the blood, removal of waste products of metabolism and serve homeostatic functions. They are located in the abdominal cavity, more specifically in the paravertebral gutter and lie in a retroperitoneal position at a slightly oblique angle. There are two kidneys on each side, and the asymmetry

within the abdominal cavity caused by the position of the liver, typically results in the right kidney being slightly lower and smaller than the left [1]. Each adult kidney weighs between $125 \mathrm{~g}$ and $170 \mathrm{~g}$ in male; and between $115 \mathrm{~g}$ and $155 \mathrm{~g}$ in females [2].Coffee, also known as café with botanical name Arabica coffea, is one of the most frequently consumed beverages. It contains a number of potentially biologically active constituents including non-volatile alkaloids and non-volatile chlorogenic acid. Coffee has the highest and most variable caffeine content (1,3,7- trimethylxanthine), it is also a rich source of phenolic compounds which have been shown to have antioxidant properties. Renal failure is an abrupt or rapid decline in renal filtration function often marked by a rise in serum creatinine concentration, or a rise in blood urea nitrogen [3]. Habitual coffee consumption has been reported to be associated with increased estimated glomerular filtration rate determined on serum creatinine levels [4]. It has also been shown that chronic coffee consumption has adverse renal effects in neuropathy associated with high blood pressure; as a result, a switch from caffeinated to decaffeinated coffee has been necessitated [5,6].Caffeine belongs to the family of heterocyclic compounds known as purines. It has the systematic name; 3,7-dihydro-1,3,7-trimethyl-1-H-purine-2,6-Dione. It is classified as an alkaloid and its chemical formula is $\mathrm{C}_{8} \mathrm{H}_{10} \mathrm{~N}_{40}$; with a molar mass of $194.1 \mathrm{~g}$. It is soluble in water and other organic solvents. It appears in pure form as crystals and can be prepared by extraction from natural sources or synthesis from uric acid [7]. Metabolites of caffeine include three main dimethylxanthines namely: paraxanthine, theobromine, and theophyline [8]. Caffeine from coffee is absorbed by the small intestine within 45 minutes of ingestion, and is distributed throughout all tissues of the body [9]. The biological half-life of caffeine varies widely among individuals according to factors such as age, liver function, pregnancy etc. it can also be significantly altered by drug or hormonal state [10]. In healthy individuals, the half-life of caffeine is about $3-7$ hours. This range is doubled during pregnancy to about 15 hours [11]. Caffeine is metabolised in the liver by the cytochrome P450 oxidase enzyme system, particularly the CYP 1A2 isozyme; into three (3) dimethylxanthines each of which has its own effects on the body [12]. Caffeine is a central nervous system and metabolic stimulant [12] used both recreationally and medically to reduce physical fatigue, and to restore alertness when drowsiness occurs [13]. It allows for faster and clearer flow of thought and general body co-ordination [14]. The amount of caffeine needed to produce effect varies from person to person depending on body size and degree of tolerance. It also improves performance during sleep deprivation, but may lead to subsequent insomnia [15]. Decaffeinated coffee refers to any coffee that has most of the 
caffeine present in it removed via a variety of decaffeination processes. However, these processes do not make coffee caffeine free, rather, significantly reduces the amount of caffeine present. The first commercially decaffeinated coffee was produced in Bremen in the beginning of the twentieth century. The coffee was developed in 1903 by a team of researchers

led by Ludwig Roselius in Bremen, Germany. According to FDA guidelines, decaffeinated coffee must have $97 \%$ of the caffeine removed. In actuality, the caffeine content of decaffeinated coffee fluctuates. The decaffeination process does not eliminate chlorogenic acid which is the substance that serves to regulate blood glucose levels [16]. High levels of antioxidants are also present and they prevent conditions associated with aging process and cancer [17]. The polyphenols found in coffee beans are not lost and they boost the brain's cognitive abilities, thus improving memory [18]. Since caffeine has been linked to heart conditions like stroke, its removal therefore reduces the incidence of heart conditions in consumers [19]. This study was designed to compare renal histomorphological changes associated with the administration of caffeinated and decaffeinated coffee to male albino Wistar rats.

\section{Animals and Treatment}

\section{Materials And Methodology}

Thirty-five (35) male albino Wistar albino rats weighing between 135 - $185 \mathrm{~g}$ were used for the study. The rats were obtained from the animal house of the Department of Pharmacology and Toxicology, Faculty of Pharmacy, University of Uyo, Nigeria. The rats were fed with pellets and clean drinking water ad libitum. They were randomly divided into 7 groups with 5 animals per group. Group 1 served as control while the experimental groups were 2-7. The Acute toxicity dose $\left(\mathrm{LD}_{50}\right)$ of the coffee was determined to be $300 \mathrm{mg} / \mathrm{kg}$ of the animal. Caffeinated coffee at doses of $5 \mathrm{mg}, 10 \mathrm{mg}$ and $15 \mathrm{mg}$ per $\mathrm{kg}$ body weight of animal was administered orally to treatment Groups 2, 3,4 respectively; and $5 \mathrm{mg}, 10 \mathrm{mg}, 15 \mathrm{mg}$ per $\mathrm{kg}$ body weight of decaffeinated coffee was administered orally to treatment Groups 5, 6, 7. The caffeinated and decaffeinated coffee were obtained from a supermarket in Uyo Metropolis, Akwa Ibom State, Nigeria.

\section{Tissue Processing}

The coffee was administered to the experimental rats daily for 28 days, after which the animals were sacrificed under chloroform inhalational anesthesia. The kidneys of each rat were harvested and weighed immediately; and placed in $10 \%$ buffered formal saline. The preserved tissues were dehydrated with ascending grades of alcohol $(70 \%, 90 \%, 95 \%$ and $100 \%)$ one hour per each concentration. The tissues were then cleared in two changes of xylene for one hour and infiltrated with molten paraffin wax at $60^{\circ} \mathrm{C}$, two changes for an hour. The tissues were further embedded in molten paraffin and sectioned at $5 \mu \mathrm{m}$ using a rotary microtome. The tissue sections were then stained using Haemotoxylin and Eosin. Light microscopic examinations were taken and presented as plates.

\section{Statistical Analysis}

Statistical analysis was performed using Microsoft SPSS application; analysis of variance (ANOVA) and Least Significant Difference (LSD) post hoc multiple comparison test was perform. Experimental data was presented as Mean \pm Standard Deviation $(\mathrm{SD})$. Values of $(P<0.05)$ were considered to be statistically significant.

\section{Results}

Table 1:Effects of Caffeinated and Decaffeinated Coffee on Body Weight of Male Albono Wistar Rats

\begin{tabular}{|l|l|}
\hline Group & Body Weight \\
\hline Group 1 CONTROL & $0.46 \pm 0.16$ \\
\hline Group 2 (CAF-LD) & $0.80 \pm 0.20$ \\
\hline Group 3 (CAF-MD) & $0.88 \pm 0.22$ \\
\hline Group 4 (CAF-HD) & $1.2 \pm 0.05$ \\
\hline Group 5 (DEC-LD) & $0.88 \pm 0.16$ \\
\hline Group 6 (DEC-MD) & $1.6 \pm 0.13$ \\
\hline Group 7 (DEC-HD) & $1.1 \pm 0.09$ \\
\hline
\end{tabular}

\section{Effects of Caffeinated and Decaffeinated Coffee on the Histology of the Rat's Kidney}

Histological section of the Kidney without treatment at magnification $\mathrm{x} 400$ (Plate 1) revealed normal cellular cytoarchitecture of proximal and distal convoluted tubules, collecting ducts, renal corpuscle containing glomerulus and lined with squamous epithelial within normal cellular architecture. Histological section of the kidney treated with $5 \mathrm{mg} / \mathrm{kg}$ (low dose) caffeinated coffee (Plate 2) revealed onset of tangential tubular degeneration, loss of epithelial lining and vascular degeneration as compared to control group. Histologic section of the kidney treated with $10 \mathrm{mg} / \mathrm{kg}$ (middle dose) caffeinated coffee (Plate 3) showed tubular degeneration, loss of epithelial lining and vascular degeneration as compared to control group. 
Histological section of the kidney treated with $15 \mathrm{mg} / \mathrm{kg}$ (high dose) of caffeinated coffee (Plate 4) indicating tubular degeneration, loss of epithelial lining and vascular degeneration as compared to control group. Histologic section of the kidney treated with $5 \mathrm{mg} / \mathrm{kg}$ (low dose) of decaffeinated coffee at magnification $\mathrm{x} 400$ (Plate 5) revealed slight area of glomerular inflammation as s compared to control group. Histological section of the kidney treated with $10 \mathrm{mg} / \mathrm{kg}$ (middle dose) decaffeinated coffee (Plate 6) showed slight area of glomerular inflammation as s compared to control group. Histological section of the kidney treated with $15 \mathrm{mg} / \mathrm{kg}$ of (high dose) decaffeinated coffee (Plate 7) revealed slight area of glomerular inflammation as $\mathrm{s}$ compared to control group

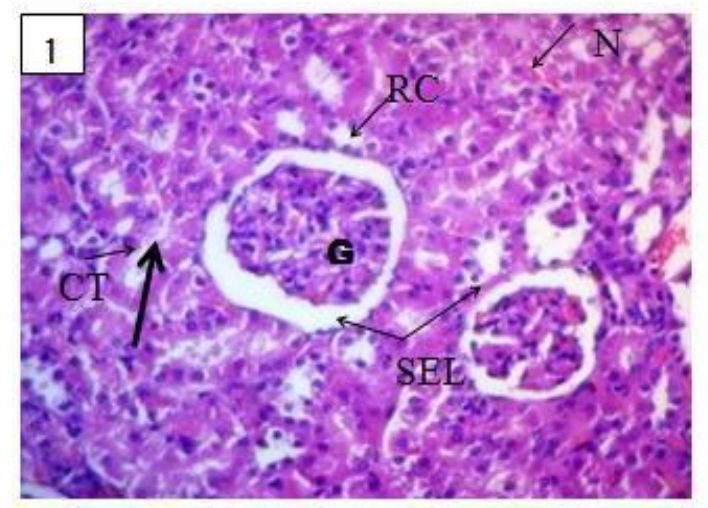

Plate 1: Photomicrographs of rat kidney without treatment at magnification $\mathrm{x} 400$ stained with $\mathrm{H} \& \mathrm{E}$ method. Keys: Renal corpuscle (RC) Convoluted tubules (CT), Collecting ducts (CD), Squamous epithelial lining (SEL), Glomerulus (GL) and Nucleus (N).

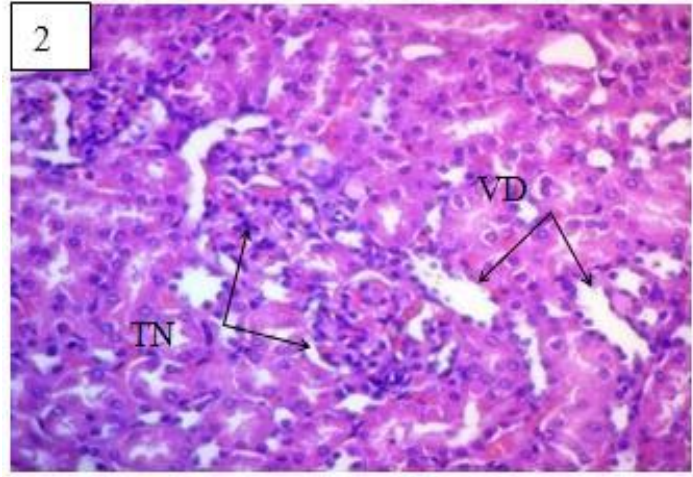

Plate 2: Photomicrographs of Kidney treated with $5 \mathrm{mg} / \mathrm{kg}$ of caffeinated coffee (low dose) at magnification $\mathrm{x} 400$ stained with H\&E method. Keys: Tubular necrosis (TN), Vascular degeneration (VD).

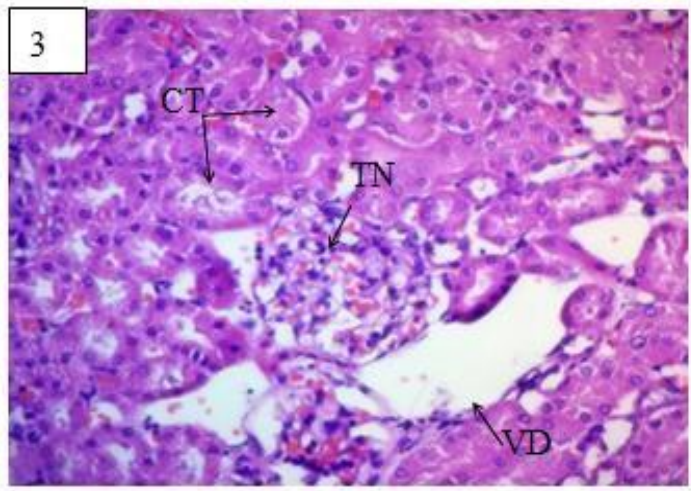

Plate 3: Photomicrographs of Kidney treated with $10 \mathrm{mg} / \mathrm{kg}$ of caffeinated coffee (Middle dose) at magnification $\mathrm{x} 400$ stained with H\&E method. Keys: Convoluted tubules (CT), Tubular necrosis (TN), Vascular degeneration (VD).

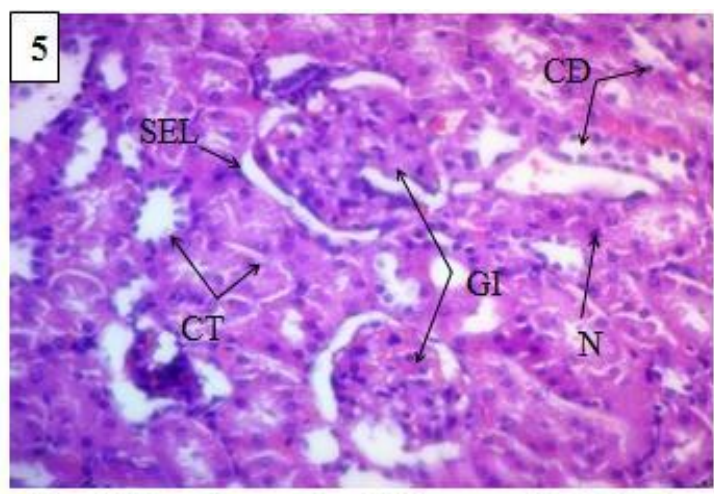

Plate 5: Photomicrographs of Kidney treated with $5 \mathrm{mg} / \mathrm{kg}$ of Decaffeinated coffee (low dose) at magnification $\times 400$ stained with H\&E method. Keys: Convoluted tubules (CT), Collecting ducts (CD), Squamous epithelial lining (SEL), Glomerulus (Gl) and Nucleus (N).

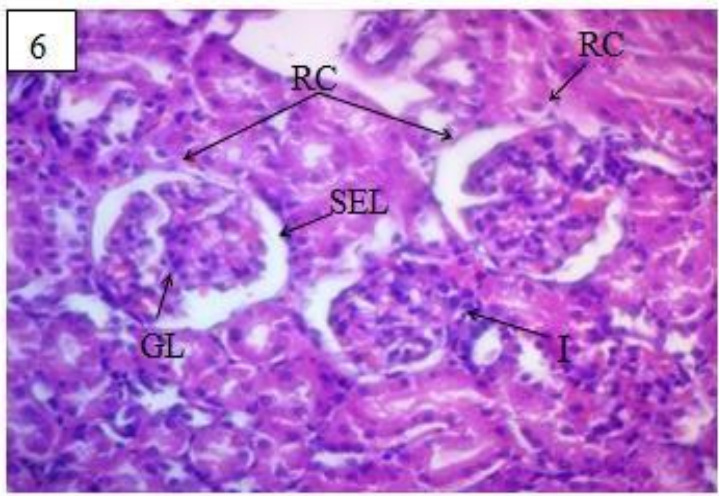

Plate 6: Photomicrographs of Kidney treated with $10 \mathrm{mg} / \mathrm{kg}$ of Decaffeinated coffee (middle dose) at magnification $\times 400$ stained with H\&E method. Keys: Renal corpuscle (RC), Squamous epithelial lining (SEL), Glomerulus (GL), Inflammation (I). 


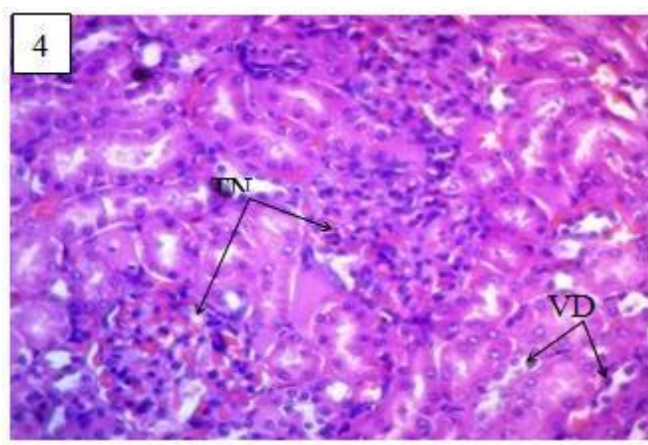

Plate 4: Photomicrographs of Kidney treated with $15 \mathrm{mg} / \mathrm{kg}$ of caffeinated coffee (High dose) at magnification $\mathrm{x} 400$ stained with H\&E method. Keys: Tubular necrosis (TN), Vascular degeneration (VD).

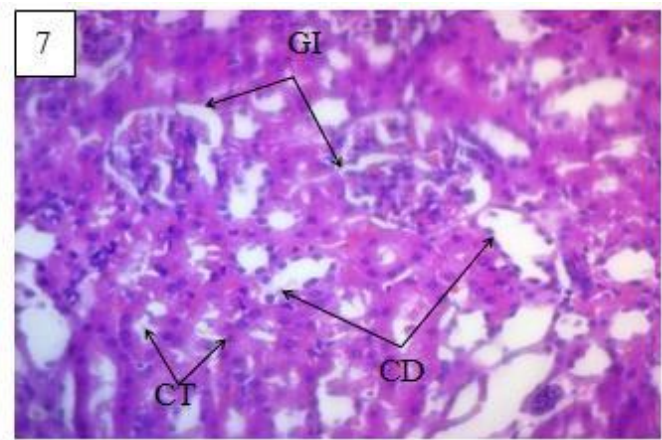

Photomicrographs of Kidney treated with $15 \mathrm{mg} / \mathrm{kg}$ of Decaffeinated coffee (high dose) at magnification $\mathrm{x} 400$ stained with H\&E method. Keys: Convoluted tubules (CT), Collecting ducts (CD), Glomerulus (GL),

\section{Discussion}

The weight gain seen in the groups treated with doses of decaffeinated coffee was higher than in those treated with caffeinated coffee. This observation supports a study that showed that coffee may actually promote weight gain by stimulating cortisol production. Cortisol is a stress hormone that has a major role in facilitating the fight or flight mechanism. Under stress, cortisol provides the body with glucose by utilizing protein stores via gluconeogenesis in the liver [20]. Elevated cortisol level consistently produces glucose leading to increased blood sugar levels along with insulin suppression which causes the cells to be starved of glucose [21]. These cells then send hunger signals to the brain where appetite is influenced by binding to hypothalamus receptors in the brain. Elevated cortisol levels also lead to weight gain via visceral fat storage and mobilization of triglycerides.

The histopathological findings of this study indicated that coffee consumption for 28 days exacerbated renal cyto-architectural alterations thereby inducing severe tubule-interstitial and glomerular damage in male albino Wistar rats. The histological section of kidney tissue without treatment (Plate 1) revealed that the normal cellular profile of the proximal and distal convoluted tubules, collecting duct, renal corpuscle (containing glomerulus) remained unaltered [22,23].

The kidney tissue treated with low dose $5 \mathrm{mg} / \mathrm{kg}$ (Plate 2 and 5) showed an onset of tubular degeneration, loss of epithelial lining and vascular degeneration as compared to the control. Plates 3 and 4 of kidney treated with middle dose $10 \mathrm{mg} / \mathrm{kg}$ and high dose $15 \mathrm{mg} / \mathrm{kg}$ of caffeinated coffee respectively also revealed abnormal cellular pattern, and tubular degeneration as compared to the control. However, the degenerative effects of the tubules were more severe at high dose when compared to the control. The kidney tissues seen in Plates 5-7, all showed slight area of glomerular inflammation. This suggests that the administration of decaffeinated coffee had minimal effect in contrasts to caffeinated coffee administration which posed a more severe risk on the cytoarchitecture of the kidney. The administration of low dose caffeinated coffee revealed an onset of tubular and vascular degeneration [24], as well as a loss of epithelial lining but increased dose poses a higher risk on renal function.

The result obtained from this study showed that caffeinated and decaffeinated coffee consumption exhibits adverse effect on the cytoarchitecture of the kidney of male albino Wistar rats. The study also showed that there was a gain in body weight of all animals, suggesting the ability of caffeine consumption to potentiate and accelerate weight increase. Long-term caffeine consumption may have adverse effects on renal function hence chronic consumption in high quantity of caffeinated coffee should be discouraged or reduced.

\section{Ethical Issues}

The approval for the study and the use of animals was obtained from the Faculty Animal Care and Use Committee and Research and Ethical Committee of the Faculty of Basic Medical Sciences of University of Uyo, Nigeria.

\section{References}

[1]. Glodny, B., Unterholzner, V., Taferner, B. (2009). Normal kidney size and its influencing factors.

[2]. Walter, F. (2004). Medical Physiology: A cellular and molecular approach. Elsevier/Saunders.

[3]. Nakajima, K., Hirose, K., Ebata, M., Morita, K., Munakata, H. (2009). Association between habitual coffee consumption and normal or increased estimated glomerular filtration rate in apparently healthy adults. British journal of nutrition, 103:149-152.

[4]. Kotani, K., Sakane, N., Yamada, T., Taniguchi, N. (2010). Association between habitual coffee consumption and the estimated glomerular filtration rate in the general Japanese population: preliminary data regarding c-reactive protein concentration. 48: 17731776. 
[5]. Cano-Marquina, A., Tarin, J.J., Cano, A. (2013). The impact of coffee on health. National centre for biotechnology information, 1:7-21.

[6]. Lamarine, R.J. (1994). Selected health and behavioural effects related to the use of caffeine. Journal of community health, 19:44964966.

[7]. Central Coffee Research Institute. (2012). Coffee research station. Karnataka, india.

[8]. Bennett, A.W., Bonnie, K.B. (2001). The world of caffeine: the science and culture of the world's most popular drug. Journal of medicine, 344:1256.

[9]. Liguori, A., Hughes, J.R., Grass, J.A. (1997). Absorption and subjective effects of caffeine from coffee, cola and capsules. National center for biotechnology information, 3:721-6.

[10]. Brodbaek, H.B., Damkier, P. (2007). The treatment of hyperemesis gravidarum with chlorobutanol-caffeine rectal suppositories in Denmark: Practice and evidence. New York: Times books.

[11]. Fredholm, B.B., Battig, K., Holmen, J., Nehlig, A., Zuartau, E.E. (1999). Actions of coffee in the brain with special reference to factors that contribute to its widespread use. National centre for biotechnology information, 1:83-133.

[12]. Nehlig, A., Daval, J.L., Debry, G. (1992). Caffeine and the central nervous system: mechanisms of action, biochemical, metabolic and psychostimulant effects. National center for biochemical information, 1:139-170.

[13]. Bolton, S. (1981). Caffeine: psychological effects, use, and abuse. Journal of orthomolecular psychiatry, 3:202-211.

[14]. Ukers, W. (1935). All about coffee. New York: The tea and coffee trade journal company.

[15]. Snel, J., Lorist, M.M. (2011). Effects of caffeine on sleep and cognition. National Centre for biotechnology information, 190:105117.

[16]. Van-Dam, R.M. (2008). Coffee consumption and risk of type 2 diabetes, cardiovascular diseases and cancer: Applied physiology, nutrition, and metabolism. pp.1269-1283.

[17]. Arab, L. (2010). Epidemiologic evidence on coffee and cancer. National center for biotechnology information, 3: $271-283$.

[18]. Graham, J.R. (1954). Rectal use of ergotamine tartrate and caffeine alkaloid for the relief of migrane. The new England journal of medicine, 250:936-8.

[19]. Ding, M., Bhupaathiraju, S.N., Satija, A., Van-Dam, R.M. (2014). Long term coffee consumption and risk of cardiovascular disease: a systematic review and a dose-response meta-analysis of prospective cohort studies. American heart association, 129:64359.

[20]. Newton, R., Broughton, L.J., Lind, M.J., Morrison, P.J., Rogers, H.J., Bradbrook, I.D. (1981). Plasma and salivary pharmacokinetics of caffeine in man. National center for biotechnology information, 1:45-52.

[21]. Meyers, H. (2007). Suave molecules of mocha: coffee, chemistry, and civilization. Clinical nutrition and metabolic care, 6:745751 .

[22]. Wein, A.J., Kavoussi, L.R., Novick, A.C., Partin, A.W., Peters, C.A. (2007). Urology (9 ${ }^{\text {th }}$ Edition). International journal of urological nursing, 1: 24.

[23]. Moore, K.L., Dalley, A.F., Agar, A.M. (2006). Clinically oriented anatomy (5 ${ }^{\text {th }}$ Edition). Baltimore: Lippincott Williams and Wilkins.

[24]. Cavendish, M. (2008). Kidney disorders: disease and disorders. Tarrytown, New York: Marshall Cavendish corporations. 